\title{
28 Research Square \\ Experimental Study On The Influence of Mental Fatigue On Risk Decision Making of Miners
}

\author{
Aifang Jia ( $541737353 @ q q . c o m$ ) \\ Xi'an University of Science and Technology \\ xinyue Guo \\ Southwest University \\ Shuicheng Tian \\ Xi'an University of Science and Technology
}

\section{Research Article}

Keywords: mental, decision-making, fatigue, work.

Posted Date: September 20th, 2021

DOI: https://doi.org/10.21203/rs.3.rs-834958/v1

License: (c) (1) This work is licensed under a Creative Commons Attribution 4.0 International License. Read Full License 


\section{Abstract}

Mental fatigue increases risk-taking behavior. Using data collected between June 15 and August 6, 2020, this study investigates the impact of miners' mental fatigue on risk decision-making to improve risk prevention and prediction abilities, and to reduce the occurrence of coal mine safety accidents. A total of 274 and 33 people participated in the preliminary and formal experiments, respectively. The participants, who were coal miners, visited the lab thrice to complete the pre-experiment, Balloon Analog Risk Task (BART), lowa Gambling Task (IGT). On the BART, mental fatigue displayed a significantly positive association with risk preference. On the IGT, as mental fatigue increased, net scores continuously decreased, while the frequency of making unfavorable decisions and the probability of taking risks increased. The BART value had no or weak correlations with the net score. Results suggest that mental fatigue leads to an increasing propensity to take risks. Therefore, regarding coal mine safety management, further attention is necessary concerning miners' mental health, addressing mental fatigue, increasing rest time, and reducing night work. Furthermore, reasonable diet, improved working environments, and a positive attitude toward work should be promoted to reduce or eliminate mental fatigue and avoid decision-making errors that could cause accidents.

\section{Introduction}

It has been reported that $97 \%$ of mine accidents in China are caused by people ${ }^{1}$. This is largely attributable to decision-making errors resulting from mental fatigue. As coal mines become increasingly mechanized, various kinds of safety technologies are being adopted. Accordingly, fewer accidents are relatively uncontrollable. However, the proportion of coal mine accidents attributable to human error is increasing. Accidents such as the coal and gas outburst in Guanglong Coal Mine on December 17, 2019; major gas explosion in Pingyao City on November 18, 2019; and the Ningwu County Coal Mine roof collapse on June 19, 2019 are some examples. Therefore, to prevent human-caused accidents in coal mines, it is important to study the impact of miners' mental fatigue on their risky decision-making.

Grandjean introduced the concept of mental fatigue ${ }^{2}$, with further definition provided by Thiffault et al. ${ }^{3}$. Mental fatigue can be described as reduced motivation caused by factors such as lethargy and cognitive impairment $4,5,6,7,8$ resulting from heavy mental work, excessive nervous system tension, or long durations of monotonous and tedious work $9,10,11,12,13,14$. Mental fatigue leads to reduced goal-directed attention and flexibility of behavioral responses, as well as an increase in automatic behavior ${ }^{15}$, negatively impacting the training time required to achieve manual dexterity ${ }^{16}$. Mental fatigue affects attention, making people less able to suppress irrelevant information as well as behavioral responses based on such information, thereby reducing the accuracy of responses ${ }^{17}$. Mental fatigue has greater impacts in specific situations. The literature has shown that it negatively affects endurance performance ${ }^{18}$, increases the perceived need for physical tasks ${ }^{19}$, and hinders football performance ${ }^{20}$, among others. The 
Balloon Analog Risk Task (BART) and the lowa Gambling Task (IGT) are important tools for assessing decision-making 21,22,23,24,25. Research shows that mental fatigue can make risky decision-making more conservative $26,27,28,29,30,31,32$.

It should be noted all of these aforementioned studies employed laboratory-induced mental fatigue and were conducted over the short term. Therefore, how does mental fatigue at work affect risk decisionmaking? Very little research has been done, particularly on the effects of mental fatigue in miners on risky decision-making. This paper examines mental fatigue and miners' risk decision-making, adopts a scientific research concept, makes full use of the research theories of related disciplines, including various analytical tools and methods, and preliminarily demonstrates the effect of mental fatigue on miners' risk decision-making.

\section{Materials And Methods}

\section{Participants.}

The preexperiment participants: On June 15, 2020, 274 employees of a coal mine in Gaoping, Shanxi, who frequently were required to work underground were invited to participate in this study. all with informed consent using procedures approved by Research Ethics Committee, Institute of Safety Management and Risk Control, Xi 'an University of Science and Technology(Grant No:XUST2020058006). The coal mine has 575 employees, of which 274 work underground often or exclusively. Therefore, only 274 people were invited to participate. All 274 questionnaires distributed were retrieved; however, one employee who randomly answered questions was excluded. Thus, 273 valid questionnaires were obtained.

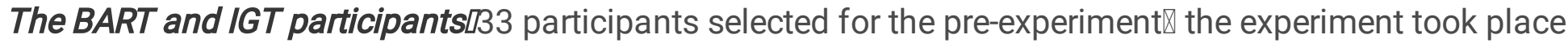
in a quiet training room in the Shouyang Coal Mine in Gaoping, Shanxi Province from July 28 to August 6 , 2020. The participants were required to have obtained adequate rest and food the previous day and were not allowed to overeat, drink, or consume caffeine. After arriving in the training room, the participants first read and signed the informed consent form. They were informed that they would be paid for their participation in the form of $20 \%$ of the prize money obtained in the game. Afterward, they completed the questionnaire. Then, the participants attempted the BART and IGT on a Lenovo laptop. After completing the task, they received $20 \%$ of the reward they earned in the experiment.

All participants had normal or corrected-to-normal vision, reported no history of neurological or psychiatric disorders. The study was approved by the Academic Committee of Xi 'an University of Science and Technology (which has the function of managing academic affairs. It has the right to approve the rationality and ethics of the experimental design.), All experiments were performed in accordance with the relevant guidelines All participants gave written informed consent prior to the experiment and were reimbursed for the time they spent at the study site. 


\section{Experimental task.}

\section{The pre-experiment:}

The questionnaire used by the participants was derived from the Maslach Burnout Inventory-General Survey (MBI-GS) ${ }^{34}$. The MBI-GS examines emotional exhaustion, low sense of accomplishment, and dehumanization, using 22 items. rated on a five-point Likert scale, from 5 (never occurred) to 1 (always is the case); Using Kalimo et al. 's ${ }^{33}$ calculation method, the higher the score, the more serious the mental fatigue. Cronbach's alpha was 0.88 in the current study.

\section{BARTDthe Balloon Analog Risk TaskJ:}

The The BART is a computerized decision-making task developed by Lejuez et al. ${ }^{35,36}$ to assess the propensity to make risky decisions. It has been applied in several domestic and international studies in the field of decision-making 37,38,39 This study used a self-edited Chinese version of the balloon simulation task.

Participants performed the BART on a Lenovo desktop computer (17-inch screen) from July 28 to 30 , 2020. all with informed consent using procedures approved by the Academic Committee of Xi 'an University of Science and Technology. They were asked to inflate 30 balloons in a computer simulation. A balloon expanded each time it was clicked, with the participant awarded 0.2 yuan for each click. Each balloon could be inflated for up to 30 clicks but would explode after a randomly chosen fixed number of clicks, from 1-30 (this process was computer-automated). If the balloon exploded, the payoff was 0 yuan. Participants had to decide whether to keep inflating a balloon or stop inflating the balloon and take the money they had earned up until that point. To inflate, they pressed the $\mathrm{J}$ key, while to collect the money obtained for that balloon, they pressed the F key. Variables measured consisted of the total number of inflations, the total number of balloons that exploded $(0-30)$, and total number of unexploded balloons. The BART value consisted of the total number of inflated balloons/the number of unexploded balloons; the experiment featured rewards, not punishments. At the end of the BART experiment, participants received $20 \%$ of the prize money they earned in the game.

\section{IGTDlowa Gambling Task]:}

The IGT is a laboratory task that simulates real decision-making situations. It is an evaluation tool developed by Bechara et $\mathrm{al}^{40}$. to measure risky decision-making that has been applied in various studies in the field of decision-making ${ }^{41,42}$

Participants performed the IGT on a Lenovo desktop computer (17-inch screen) from August 4 to 6, 2020. all with informed consent using procedures approved by Research Ethics Committee, Institute of Safety Management and Risk Control, $\mathrm{Xi}$ 'an University of Science and Technology. They were instructed to choose a total of 100 cards from four decks on the computer screen (A, B, C, or D) in each round to earn 
as much money as possible and reduce losses; some decks are better choices than others. The variables were as follows: advantageous card choices (0-100), unfavorable card choices (0-100), net score (the number of advantageous cards - the number of unfavorable cards), and total revenue earned. The initial capital per participant was 20 yuan, and the task flagged poor choices. If the participant chose a card from deck $A$, they would receive a profit of -1 yuan each time, but five cards out of 10 would include a bonus ranging from 1.5 to 3.5 yuan, which would leave them with 12.5 yuan in total. If the participant chose deck B, they would lose 1 dollar each time, but one out of 10 cards would include a 12.5-yuan bonus. In deck $C$, the participant would receive a profit of -0.5 yuan each time, but five cards out of 10 would include a bonus of 0.25 to -0.75 yuan, which would leave them with 2.5 yuan in total. When choosing from deck $D$, the participant would get -0.5 yuan each time, but one out of 10 cards would include a bonus of 2.5 yuan. Therefore, in the long run, $A$ and $B$ were the better decks, while $C$ and $D$ reflected poor choices. Before the experiment, the participants were unaware of which decks were favorable. They were only told that some of the decks were favorable while some were unfavorable, and that they had to choose favorable cards to obtain the maximum profit.

\section{Questionnaire:}

As risk decision-making is affected by risk preference, self-control ability, and emotion, experimental data were collected through the Miners' Risk Preference Scale, Brief Self-Control Scale (BSCS), and the Positive and Negative Affect Schedule (PANAS).

\section{Miners' Risk Preference Scale:}

The risk preference index was evaluated using the Miners' Risk Preference Scale prepared by Professor HongXia Li et al. ${ }^{43}$, which includes 14 items. Risk is divided into cognitive preference, emotional risk preference, and three dimensions of behavioral intention preference, rated on a five-point Likert scale, from 5 (never occurred) to 1 (always is the case); the higher the score, the lower the willingness to take risks. Cronbach's alpha was 0.93 in the current study.

\section{BSCS:}

The BSCS, compiled by Tangney and Baumeisterv ${ }^{44}$ and revised by Tan and Guo ${ }^{45}$, was used as a screening tool for trait self-control. It consists of 13 questions divided into five dimensions, namely, overall self-discipline, impulse control, healthy habits, resistance to temptation, and reliability. The items are rated on a five-point Likert scale from 1 (completely inconsistent) to 5 (completely consistent). The higher the total score, the higher the level of individual trait self-control. Cronbach's alpha was 0.79 in the current study.

PANAS:

The Chinese version of Watson et al.'s ${ }^{46}$ PANAS was adopted. The scale has been observed to be consistent across cultures ${ }^{47}$; thus, it could be used to assess the emotional self-rating of Chinese miners. 
The scale is composed of 20 adjectives describing emotions, with participants asked to evaluate their emotions on a five-point Likert scale from 1 (almost none) to 5 (various). Positive and negative emotions are separated into two parts. A high score on positive emotion indicates that an individual is energetic and happy, while a low score indicates indifference. On the contrary, a high score on negative emotion indicates subjective confusion and a painful emotional state, while a low score indicates calmness. Cronbach's alpha was 0.78 in the current study.

\section{Experimental procedures:}

For the selected participants, the experiment took place in a quiet training room in the Shouyang Coal Mine in Gaoping, Shanxi Province from July 28 to August 6, 2020. The participants were required to have obtained adequate rest and food the previous day and were not allowed to overeat, drink, or consume caffeine. After arriving in the training room, the participants first read and signed the informed consent form. They were informed that they would be paid for their participation in the form of $20 \%$ of the prize money obtained in the game. Afterward, they completed the questionnaire. Then, the participants attempted the BART and IGT on a Lenovo laptop. After completing the task, they received $20 \%$ of the reward they earned in the experiment.

\section{Analysis process}

SPSS 25.0 (IBM Corp, Armonk, NY, USA), Microsoft Excel 19.0, and Origin 2018 (OriginLab, USA) were used to analyze the data. A single factor analysis of variance (ANOVA) was conducted on demographic variables and emotion to exclude the influence of demographic variables and emotion on the mental fatigue effect. Regression analysis was conducted between additional variables and BART value and net score to eliminate the interference and influence of non-research factors such as emotion, trait selfcontrol, risk preference, and other additional variables on the experiment. The BART value and covariate, net score and covariate of different mental fatigue degree were analyzed by repeated measurements variance.

\section{Results}

\section{the pre-experiment Analysis}

SPSS 25.0 (IBM Corp, Armonk, NY, USA) and Microsoft Excel 19.0 were used for the analysis. We followed Kalimo et al.33, who applied the weighted scoring method, that is, the score of mental fatigue $=0.40 \times$ emotional exhaustion $+0.30 \times$ dehumanization $+0.30 \times$ low sense of achievement. Through this analysis, we were able to select 22 participants with mild mental fatigue (mental fatigue scores $\in[1$, $1.49]), 22$ with moderate mental fatigue (mental fatigue scoring $\in[2.53,2.63]$ ), and 22 with severe mental fatigue (mental fatigue scores $\in[3.41,4.38]$ ). Subsequently, we randomly selected 11 participants from these candidates according to their mental fatigue levels(Eleven participants were selected for each level 
of mental fatigue), enrolling 33 participants in the formal experiment. The remaining 33 participants took part in another experiment that ran simultaneously. The inclusion criteria were: regularly working underground in a coal mine, being right-handed, and having normal vision or corrected visual acuity. (Fig. 1).After excluding three participants who were not serious about the experiment, we obtained experimental data for 30 participants.

\section{Descriptive statistical analysis of demographic variables}

The 30 participants were male, right-handed, with normal or corrected visual acuity and no neurological disease. Participant ages were as follows: 10 participants were aged $24-30$ years (33.33\%), 16 were aged 31-40 (56.7\%), and four participants were aged 41 or above (13.33\%). Regarding education level, 14 people had technical secondary school education or below $(46.7 \%), 12$ had junior college education $(40 \%)$, and four had undergraduate degrees (13.3\%). Regarding job role, there were 24 normal workers $(80 \%)$ and cadres six $(20 \%)$. Nineteen participants $(63.3 \%)$ had worked for $0-5$ years; nine participants had worked 6-10 years (30\%), and two had worked for more than 11 years $(6.7 \%)$. Regarding years of working underground, 21 people had worked for $0-5$ years $(70 \%)$ and nine for $6-10$ years $(30 \%)$. Statistical analysis of participants' demographic variables (Table 1) revealed no significant relationship between mental fatigue and age. However, the higher the education level and the greater the job responsibility, the higher the degree of mental fatigue. Further, increased mental fatigue was associated with more years worked and years of working underground.

The effect of mental fatigue did not significantly differ by age: $F(3,30)=1.325, P=0.282$; educational level: $F(3,30)=1.969, P=0.159$; job role: $F(3,30)=1.125, P=0.339$; years of working: $F(3,30)=1.385, P$ $=0.267$; and rural and urban birth: $F(3,30)=0.22, P=0.804$. However, the effect of mental fatigue significantly differed by the number of years working underground: $F=3.745, P=0.037<0.05$. Therefore, the experience of the three groups (mild, moderate, and severe mental fatigue) was not significant in terms of demographic variables, such as age, educational level, job, working years, and birth in urban or rural areas, which undermines the influence of these factors on mental fatigue. However, the three groups of subjects differed significantly in terms of years working underground; thus, this variable was potentially related to mental fatigue. Further analysis was conducted of whether years working underground were correlated with the BART value and net score (Fig. 2).

\section{Analysis of emotion scale}

The data gathered on emotions was meant to eliminate the interference of emotion as a factor. A single factor ANOVA test for positive mood showed that the mild mental fatigue group (mean $[M]=29.5$, standard deviation $[S D]=6.311)$, moderate mental fatigue group $(M=31.3, S D=7.47)$, and severe mental fatigue group $(M=29.1, S D=8.40)$ showed neither significant positive emotional experiences, $F$ 
$(3,30)=0.248, P=0.782$, nor negative emotional experiences $(M=18.8, S D=5.12 ; M=23.8, S D=3.65$; $M=21.6, S D=6.58$, for each group, respectively). Thus, the influence of emotions on mental fatigue was excluded: $F(3,30)=2.274, P=0.122$.

\section{Analysis of BART values}

\section{Descriptive statistical analysis of BART values}

Fig. 3 shows that with the increase of mental fatigue and the number of inflations and exploded balloons, the BART value gradually increases; the number of missed shots tends to decrease gradually. This suggests that with increased mental fatigue, the participants' tendency to take risks is more obvious.

Descriptive statistical analysis of BART values for participants with mild, moderate, and severe mental fatigue including total number of inflations, total number of exploded balloons, total number of unexploded balloons, and BART values is exhibited in Table 2. The BART value is Mmild $<$ Mmoderate < Msevere, that is, with the increase of mental fatigue, participants are more inclined to take risks

\section{Correlation analysis between additional variables and BART values}

We conducted correlation analysis between the four factors and BART values to exclude the interference and influence of non-research factors such as emotion, risk preference, trait self-control, and underground working years. The results are shown in Table 3. Trait self-control $(R=0.390, P<0.05)$, risk preference $(R$ $=0.334, \mathrm{P}<0.05)$, and positive emotion scores $(R=0.353, \mathrm{P}<0.05)$ were significantly correlated with BART values $(R=0.390, P<0.05)$. Therefore, for the BART, risk preference, trait self-control, and positive emotion score were incorporated into the equation as covariables for analysis (Table 3 ).

\section{Influence of mental fatigue on risk decision-making tendency}

We found that BART values in the group with severe mental fatigue $(M=10.3, S D=2.34)$ were greater than those in the moderate mental fatigue group $(M=8.65, S D=2.81)$, which, in turn, were greater than those in the mild mental fatigue group $(M=6.19, S D=2.12)$ (Fig. 4).

To further study the effect of mental fatigue on risk propensity in decision- making, we performed an ANOVA with the mental fatigue groups as the independent variables; BART values as the dependent variable; and trait self-control, willingness to take risks, and working for a fixed number of years as covariates. The results showed that the difference in BART values between groups was significant ( $F(3$, $30)=4.142, \mathrm{P}<0.05)$. The bar chart $(95 \%$ confidence interval) of BART values of different groups is shown in Fig. 4. 
Overall, the results suggest that mental fatigue has a significant effect on risk propensity in risky decision-making. The higher the level of mental fatigue, the more risk-taking behaviors (the higher the reward) and the higher the risk-seeking tendency of the participants in the BART.

\section{IGT analysis}

\section{Number of card choices}

In the IGT, high- and low-frequency reward decks contained low- and high-frequency penalty cards, respectively. Therefore, it was necessary to analyze the selection times of different types of cards by the three groups of participants to investigate their card selection characteristics. Fig. 5 shows the selection times of different types of cards by participants with different degrees of mental fatigue. As shown, with a gradual increase in mental fatigue, the tendency to choose A/B cards decreased gradually, while the tendency to choose C/D cards increased gradually.

Taking the type and class of cards as independent variables and the number of cards selected for each type as dependent variables, 2 (card type) $\times 3$ (group) mixed-design ANOVA and ANOVA were carried out. The results showed that the main effect between favorable and unfavorable cards of card type was significant: $F(2,30)=83.235, P=0.000$. The group main effect was also significant: $F(3,30)=16.789, P$ $=0.000$. As shown in Fig. 6 , with the aggravation of mental fatigue, the number of unfavorable cards gradually increased, while the number of favorable cards gradually decreased. Thus, with increasing mental fatigue, participants' risk decision-making tendency became stronger. Further, the more serious the mental fatigue, the more likely they were to take risks (Fig. 6).

\section{Statistical analysis of the net score of cards}

A repeated measures ANOVA was performed with groups as the independent variables and the net score of each decision module as the dependent variable. The results are shown in Fig. 7. The main effect between modules was significant, $F=5.944, P<0.05$, but the main effect between groups was not significant $(F=2.43, P=0.107)$. There was no significant interaction between the groups and modules, $F$ $=0.177, P=0.839$. The net scores of the participants in the mild, moderate, and severe mental fatigue groups were significantly different, as shown in Fig. 7. The overall net score of the bar graph increases with the increase in decision times. Participants' net score decreased with higher mental fatigue, reducing the overall height of the histogram. This indicates that as they experienced higher mental fatigue, participants became more inclined to take risks. 


\section{Correlation analysis between additional variables and net score}

A correlation regression analysis was conducted between the four variables and the net score to exclude the influence of non-research variables such as emotions, trait self-control, risk preference, and years of underground work on risk decision-making. The results are shown in Table 4. Positive emotion score and net score $(R=0.296, P=0.056)$ were significantly marginal. Net score had no significant correlations with negative emotion score, risk preference, trait self-control, or years working underground.

\section{Influence of mental fatigue on risk decision-making tendency}

the net score values of different groups; specifically, the net score value of the mild mental fatigue group $(M=44.5, S D=40.43)$ was greater than those of the moderate mental fatigue group $(M=23.5, S D=$ 33.08) and the severe mental fatigue group $(M=7.8, S D=38.2)$. The lower the net score, the more frequently the participants chose unfavorable cards and the greater their risk tendency, indicating that with higher mental fatigue, risk tendency increased .

To further study the effect of mental fatigue on risk propensity in decision-making, the mental fatigue groups were taken as the independent variables, with net scores as the dependent variables. The results of the regression analysis showed that the net score value differences between groups were significant ( $F$ $(3,30)=4.992, \mathrm{P}<0.05)$. This indicates that mental fatigue had a significant impact on risk-taking tendency in risk decision-making; the higher the participants' mental fatigue, the riskier their behaviors in the IGT ( more frequently choosing unfavorable cards).

\section{Comprehensive risk score}

The BART value and the IGT net score were tested by a paired samples t-test. The test results showed that $\mathrm{T}(30)=-2.32, \mathrm{P}<0.05, \mathrm{~F}=-0.283, \mathrm{P}=0.130$. There was a very significant correlation between BART values and IGT net scores. These results indicate that the BART and IGT can be used to effectively measure risk-taking tendency and provide new ideas for workers' job arrangements.

Taking mental fatigue as the control variable and BART values and IGT net scores as dependent variables, the correlation analysis showed that mental fatigue was negatively correlated with IGT net scores $(F=-0.387, P<0.05)$. In other words, the more serious the mental fatigue, the lower the IGT net score, the more frequently the participants chose unfavorable cards, and the greater the risk decisionmaking. There was also a significant positive correlation between mental fatigue and BART values $(F=$ $0.543, \mathrm{P}<0.05)$. In other words, the more severe the mental fatigue, the higher the BART value, and the greater the risk decision-making tendency.

\section{Discussion}


Previous studies have considered the effects of mental fatigue on risk-taking decisions.

However, mental fatigue in all of these prior studies was transient mental fatigue, with the studies assessing people who were currently mentally fatigued. In contrast, very few studies have considered the impact on risky decision making of mental fatigue and there has been little research on the effect of mental fatigue on miners' risky decision-making. This study focused on the effects of mental fatigue on risky decision making among front-line miners. The results showed that the coal miners studied generally exhibited mental fatigue, although this was generally moderate; fewer miners exhibited mild and severe mental fatigue. In the BART experiment, the BART values of participants increased with increasing mental fatigue, indicating that decision-making was increasingly inclined toward risk-taking. In the IGT experiment, net scores decreased with increasing mental fatigue, which indicated that participants were increasingly inclined to take risks.

In summary, increased mental fatigue was associated with an increasing tendency to make risky decisions. Therefore, it is important to regularly assess miners' psychological fatigue and risk-taking tendencies, and to arrange work schedules accordingly. To ensure the safe operation of coal mines, it is important to allocate working time and intensity in reasonable manner, reduce or avoid night shifts, and ensure workers are provided rest time. These actions would reduce the occurrence of mental fatigue, and thereby minimize unnecessary accidents and losses caused by human behavior.

\section{Conclusion}

With the increase of mental fatigue, BART value increased consistently, and participants were increasingly more inclined to take risks. In the IGT, with the increase of mental fatigue, the net score lowered accordingly, and the participants were increasingly inclined to choose from the unfavorable decks, that is, they were increasingly inclined to take risks. Based on these results, as mental fatigue increases, the risk tendency becomes increasingly stronger. In coal mine production, attention should be paid to workers' mental fatigue, reasonable allocation of working hours, and working intensity. Avoiding the accumulation of mental fatigue by reducing or eliminating night shifts and ensuring that workers get adequate rest is important for avoiding accidents and losses.

\section{Declarations}

\section{Acknowledgements $\square$}

The research was supported by the National Natural Science Foundation of China

\section{Author contributions:}

AJ contributed to the study conception and design. Material preparation, data collection and analysis were performed by AJ, ST and XG. The first draft of the manuscript was written by AJ and all authors commented on previous versions of the manuscript. All authors read and approved the final manuscript. 


\section{Funding:}

This study was supported by the National Natural Science Foundation of China (51874237).

\section{Competing interests:}

The authors declare no competing interests.

\section{Data Availability Statement:}

The data that support the findings of this study are available on request from the corresponding author, AJ. The data are not publicly available to ensure the privacy of the research participants.

\section{Ethics approval:}

This manuscript has not been published or presented elsewhere in part or in entirety.

\section{Consent to participate:}

All study participants provided informed consent.

\section{References}

1. WANG Zongxiao Experts say that $97 \%$ of China's mine accidents are caused by human activities, with 70\% of the world's deaths[J].Safety \& Health, 2011,囚17囚:34-35.

2. GRANDJEAN E. Fatigue in industry [J]. British Journal of Industrial Medicine, 1979, 36:175-186

3. THIFFAULT P B,ERGERON J.Fatigue and individual differences in monotonous Simulated driving[J].Personality And Indiv idual Differences, 2003, 34: 159-176.

4. Boksem, M, Meijman, T. F, \& Lorist, M. Efects of mental fatigue on attention: An ERP study. Cognitive Brain Research,2005;25,107-116.

5. Hopstaken, J. F, Van Der Linden, D, Bakker, A. B, \& Kompier, M. A,A multifaceted investigation of the link between mental fatigue and task disengagement. Psychophysiology,2015;52(3),305-315.

6. Lal, S. K. L, \& Craig, A. A critical review of the psychophysiology of driver fatigue. Biological Psychology, 2001;55(3),173-194.

7. Lal, S. K. L, Craig, A. Driver fatigue: Electroencephalography and psychological assessment. Psychophysiology,2002;39(3),313-321.

8. Faber,L.G.,Maurits,N.M.,\& Lorist,M.Mental fatigueafects visual selective attention [J]. PLoS ONE,2012; 7(10), $1-10$.

9. Kato, Y, Endo, H., \& Kizuka, T. Mental fatigue and impaired response processes: Event-related brain potentials in a Go/NoGo task. International Journal of Psychophysiology,2009;72(2),204-211.

10. Lin, G, Xiang, Q, Fu, X, Wang, S, Wang, S, Chen, S., et al. Heart rate variability biofeedback decreases blood pressure in prehypertensive subjects by improving autonomic function and 
barorefex[J].Journal of Alternative and Complementary Medicine(NewYork,NY),2012;18(2):143-152.

11. Melo, H. M., Nascimento, L. M., De Mello, V. O, \& Takase, E. Alpha $(8-12 \mathrm{~Hz})$ influence on reaction time in inhibitory control task. Revista Neuropsicologia Latinoamericana,2017a;9(2),33-43.

12. Ting, P, Hwang, J, Doong, J, \& Jeng, M. Driver fatigue and highway driving: A simulator study. Physiology \&Behavior,2008;94(3),448-453.

13. Wascher, E., Rasch, B., Sänger, J., Hoffmann, S, Schneider, D, Rinkenauer, G, et al. Frontal theta activity refects distinct aspects of mental fatigue. Biological Psychology,2014;96,57-65.

14. Maarten A.S. Boksem, Theo F. Meijman, Monicque M. Lorist. Effects of mental fatigue on attention: An ERP study[J].Cognitive Brain Research.2005;25 107-116.

15. MICHAEL J. DUNCAN, NICHOLAS FOWLER, OLIVER GEORGE,et al.Mental Fatigue Negatively Influences Manual Dexterity and Anticipation Timing but not Repeated High-intensity Exercise Performance in TrainedAdults[J].Researchin Sports Medicine,2015;23:1-13.

16. Marcora SM, Staiano W, Manning V (Bangor Univ, Wales,UK.Mental fatigue impairs physical performance in humans[J].Appl Physio I 2009;106:857-864.

17. Leon G. Faber,Natasha M. Maurits,Monicque M. Lorist,.Mental Fatigue Affects Visual Selective Attention[J].Mental Fatigue and Visual Attention.2012;7(10)

18. Maarten A.S. Boksem, Theo F. Meijman, Monicque M. Lorist a..Mental fatigue, motivation and action monitoring[J].BiologicalPsychology2006;72,123-132.

19. Dimitri van der Linden $\mathbb{E}$ Paul Elin(2006). Mental fatigue disturbs local processing more than global processing[J]. Psychological Research,2006;70:395-402

20. Marcora S, Staiano W. The limit to exercise tolerance in humans: mind over muscle? Eur J Appl Physiol 2010;109:763-770.

21. Mitchell R. Smith, Linus Zeuwts, Matthieu Lenoir,et al. Mental fatigue impairs soccer-specific decision making skill[J]. Journal of Sports Sciences. http://dx.doi.org/ $.1080 / 02640414.2016 .1156241$

22. Isen, A. M, \& Geva, N. The influence of positive affect on acceptable level of risk: The person with a large canoe has a large worry. Organizational Behavior and Human Decision Processes, 1987;39,145-154.

23. Isen, A. M, \& Patrick, R. The effect of positive feelings on risk taking: When the chips are down. Organizational Behavior and Human Decision Processes, 1983;31, 194-202.

24. Isen,A.M,Nygren,T.E,\&Ashby,F.G.. Influence of positive affect on the subjective utility of gain sand Iosses: Itisjustnot worththerisk. Journal of Personality and Social Psychology, 1988,55,710-717.

25. Northoff, G, Grimm, S, Boeker, H, Schmidt, C, et al.Boesiger, P. Affective judgment and beneficial decision making: Ventromedial prefrontal activity correlates with performance in the lowa Gambling Task. Human Brain Mapping,2006,27,572-587.

26. Beitz KM, Salthouse TA and Davis HP Performance on the lowa gambling task: from 5 to 89 years ofage.JExpPsycholGen 2014,143:1677-1689. 
27. Braams BR,vanDuijvenvoorde AC,Peper JS,et al. Longitudinal changes in adolescent risk-taking: a comprehensive study of neural responses to rewards, pubertal development, and risk-taking behavior. J Neurosci 2015,35:7226-7238.

28. Burdick KE, Braga RJ, Gopin CB, et al. Dopaminergic influences on emotional decision making in euthymic bipolar patients. Neuro psychopharmacology 2014,39: 274-282.

29. Verbeken S,Braet C,Bosmans G, et al.Comparing decision making in average and overweight children and adolescents[J].IntJObes 2014,38:547-551.

30. Athos TrecrocilD, Gabriele BoccolinilD, Marco Duca1,.Damiano Formentil,Giampietro Alberti.Mental fatigue impairs physical activity, technical and decision making performance during small-sided games [J].PLOSONE. 2020.

31. FENG Shen Mei..Effects of Psychological Fatigue and Framework Typeon Risk Decisions: Research from ERPs [D].Xinxiang Medical University.2018.

32. WANG LuLu囚LI Yong Juan, The Effect of Mental Fatigue and Framing on Risk Decisionmaking[J].Advances in Psychological Science,2012,20(11),1546-1550.

33. KALIMO R,PAHKIN K,MUTANEN P,et al.Staying well or burning out at work:work characteristics and personal re-sources as long-term predictors[ J].Work tress,2003,17(2):109-122.

34. MASLACH C\JACKSON SE. The measurement of experienced burnout [J]. Organ Behavior,1981,2(2):99-113.

35. LejuezCW,Jennifer P. Read and Christopher W. Kahler.et,al.Evaluation of a Behavioral Measure of Risk Taking:The Balloon Analogue Risk Task (BART). Journal of Experimental Psychology: Applied Copyright 2002 by the American Psychological Association, 2002, 89 ख2Х, 75-84.

36. Lejuez CW, Aklin WM, Zvolensky MJ, et al. Evaluation of the Balloon Analogue Risk Task (BART) as a predictor of adolescent real-world risk-taking behaviours[J].Adolesc,2003b, 26:475-479.

37. Tara L. White,C. W. Lejuez.Harriet de Wit.Test-Retest Characteristics of the Balloon Analogue Risk Task(BART)[J]. Experimental and Clinical Psycho pharmacology 2008, 565-570

38. Yuzheng ZhANG,The Effects of Emotional Intelligence and Peer Participation on Adolescents' Risk Decision-Making[D].Sichuan Normal University. 2014.

39. 44.Mengyan HE,The role of framing effects and Ego Depletion in performance on the Balloon Analogue Risk Task (BART)[D].Minnan Normal University.2017.

40. 45.Bechara A, Damasio H, Tranel D., \& Anderson, S. W. Dissociation of working memory from decision making within the human prefrontal cortex.Journal of Neuroscience the Official .Journal of the Society for Neuroscience,1998,18(1),428-437.

41. Daniel M.T. Fessler, Elizabeth G. Pillsworth, Thomas J. Flamson, Angry men and disgusted women: An evolutionary approach to the influence of emotions on risk taking[J]. Organizational Behavior and Human Decision Processes 2004, 95,107-123.

42. Shenshen Zhang, Lihong Wu, Boyu Zhang,et al. Impaired decision-making under risk in patients with functional dyspepsia[J]. Journal of Clinical and Experimental Neuropsychology. 
https://doi.org/10.1080/13803395.2020.1802406

43. LI Hong Xia,FANG Yong Bin,HAN Xiao Jing et al.LI Hong Xia,FANG Yong Bin,HAN Xiao Jing,et al.On the development and establishment of the measuring questionnaire of coal miners' risk preference level scale [J].Journal of Safety and Environment,2016,16(3):177-181.

44. Tangney,J.P,Baumeisterv R,High Self-Control Predicts Good Adjustment, Less Pathology, Better Grades, and Interpersonal Success[D].Journal of Personality, 2004.

45. TAN Shu Hua,GUO YongYu.Revision of SelfControl Scale for Chinese College Students[J].Chinese Journal of Clinical Psychology,2008;16(5):468-470.

46. Watson D,Clark L A, Tellegen A.Development and validatio no fbrief measures of positive and negative affect: ThePANAS scales.Journal of Personality and Social Psychology,1988;54(6):10631070.

47. ZHANG WeiDong, DIAO Jing. The Cross-cultural Measurement of Positive and Negative Affect Examining the Dimensionality of PANAS[J] Psychological Science 2004,27(1):77- 79.

\section{Tables}

Table 1. Descriptive statistical analysis of demographic variables

\begin{tabular}{|c|c|c|c|c|c|}
\hline & Group & $\mathrm{n}$ & M & SD & SE \\
\hline \multirow[t]{3}{*}{ Age } & Mild mental fatigue & 10 & 36.20 & 6.30 & 1.99 \\
\hline & Moderate mental fatigue & 10 & 31.90 & 5.49 & 1.73 \\
\hline & Severe mental fatigue & 10 & 32.90 & 6.69 & 2.12 \\
\hline \multirow[t]{3}{*}{ Educational level } & Mild mental fatigue & 10 & 1.40 & 0.70 & 0.22 \\
\hline & Moderate mental fatigue & 10 & 1.60 & 0.70 & 0.22 \\
\hline & Severe mental fatigue & 10 & 2.00 & 0.67 & 0.21 \\
\hline \multirow[t]{3}{*}{ Job role } & Mild mental fatigue & 10 & 1.00 & 0.00 & 0.00 \\
\hline & Moderate mental fatigue & 10 & 1.20 & 0.42 & 0.13 \\
\hline & Severe mental fatigue & 10 & 1.20 & 0.42 & 0.13 \\
\hline \multirow[t]{3}{*}{ Working for a fixed number of years } & Mild mental fatigue & 10 & 4.00 & 1.56 & 0.49 \\
\hline & Moderate mental fatigue & 10 & 6.10 & 5.17 & 1.64 \\
\hline & Severe mental fatigue & 10 & 6.40 & 2.80 & 0.88 \\
\hline \multirow[t]{3}{*}{ Years of working underground } & Mild mental fatigue & 10 & 2.80 & 3.15 & 1.00 \\
\hline & Moderate mental fatigue & 10 & 3.20 & 2.90 & 0.92 \\
\hline & Severe mental fatigue & 10 & 6.00 & 2.45 & 0.77 \\
\hline
\end{tabular}


M: mean; SD: standard deviation; SE: standard error

Table 2. Descriptive statistical analysis of BART values

\begin{tabular}{lllll} 
& Group & N & M & SD \\
\hline \multirow{3}{*}{ Total inflatable balloons } & Mild mental fatigue & 10 & 148.3 & 37.54 \\
\cline { 2 - 5 } & Moderate mental fatigue & 10 & 180.8 & 37.90 \\
\cline { 2 - 5 } & Severe mental fatigue & 10 & 196 & 20.31 \\
\hline \multirow{2}{*}{ Total exploded balloons } & Mild mental fatigue & 10 & 5.4 & 2.22 \\
\cline { 2 - 5 } & Moderate mental fatigue & 10 & 8.3 & 2.80 \\
\cline { 2 - 5 } & Severe mental fatigue & 10 & 9.8 & 3.55 \\
\hline Total unexploded balloons & Mild mental fatigue & 10 & 24.6 & 2.22 \\
\cline { 2 - 5 } & Moderate mental fatigue & 10 & 21.7 & 2.80 \\
\cline { 2 - 5 } & Severe mental fatigue & 10 & 20.2 & 3.55 \\
\hline BART value & Mild mental fatigue & 10 & 6.18 & 2.12 \\
\cline { 2 - 5 } & Moderate mental fatigue & 10 & 8.65 & 2.81 \\
\cline { 2 - 5 } & Severe mental fatigue & 10 & 10.02 & 2.34
\end{tabular}

Table 3. Correlation analysis between additional variables and BART scores

\begin{tabular}{|c|c|c|c|c|c|}
\hline & 1 & 2 & 3 & 4 & 5 \\
\hline \multicolumn{6}{|l|}{ 1. Positive emotion score } \\
\hline 2. Negative emotion score & $-0.164^{\star}$ & & & & \\
\hline 3. Risk willingness & -0.226 & $0.474 * \star \star$ & & & \\
\hline 4. Trait self-control score & -0.161 & 0.216 & 0.073 & & \\
\hline 5. Years of working underground & 0.049 & -0.076 & 0.234 & -0.275 & \\
\hline 6. BART value & $-0.353^{\star \star \star}$ & $0.093^{*}$ & $0.334^{\star \star \star}$ & $0.39 \star \star \star$ & $0.132^{\star}$ \\
\hline
\end{tabular}

Table 4. Correlation analysis between additional variables and net score 


\begin{tabular}{|llllll|}
\hline & 1 & 2 & 3 & 4 & 5 \\
\hline 1. Positive emotion score & & & & & \\
\hline 2. Negative emotion score & $-0.167^{\star}$ & & & & \\
\hline 3. Risk willingness & $-0.233^{\star}$ & $0.568^{\star \star \star}$ & & & \\
\hline 4. Trait self-control score & $-0.162^{\star}$ & $0.315^{\star \star \star}$ & $0.169^{\star}$ & & \\
\hline 5. Years working underground & $-0.204^{\star}$ & $0.131^{\star}$ & $0.265^{\star}$ & $0.13^{\star}$ & \\
\hline 6. Net score & $0.296^{\star}$ & $-0.095^{\star}$ & $-0.238^{*}$ & $-0.141^{\star}$ & $-0.138^{*}$ \\
\hline
\end{tabular}

*** $P<0.001 \quad * * P<0.05 \quad * \quad 0.05<P<0.1$

\section{Figures}

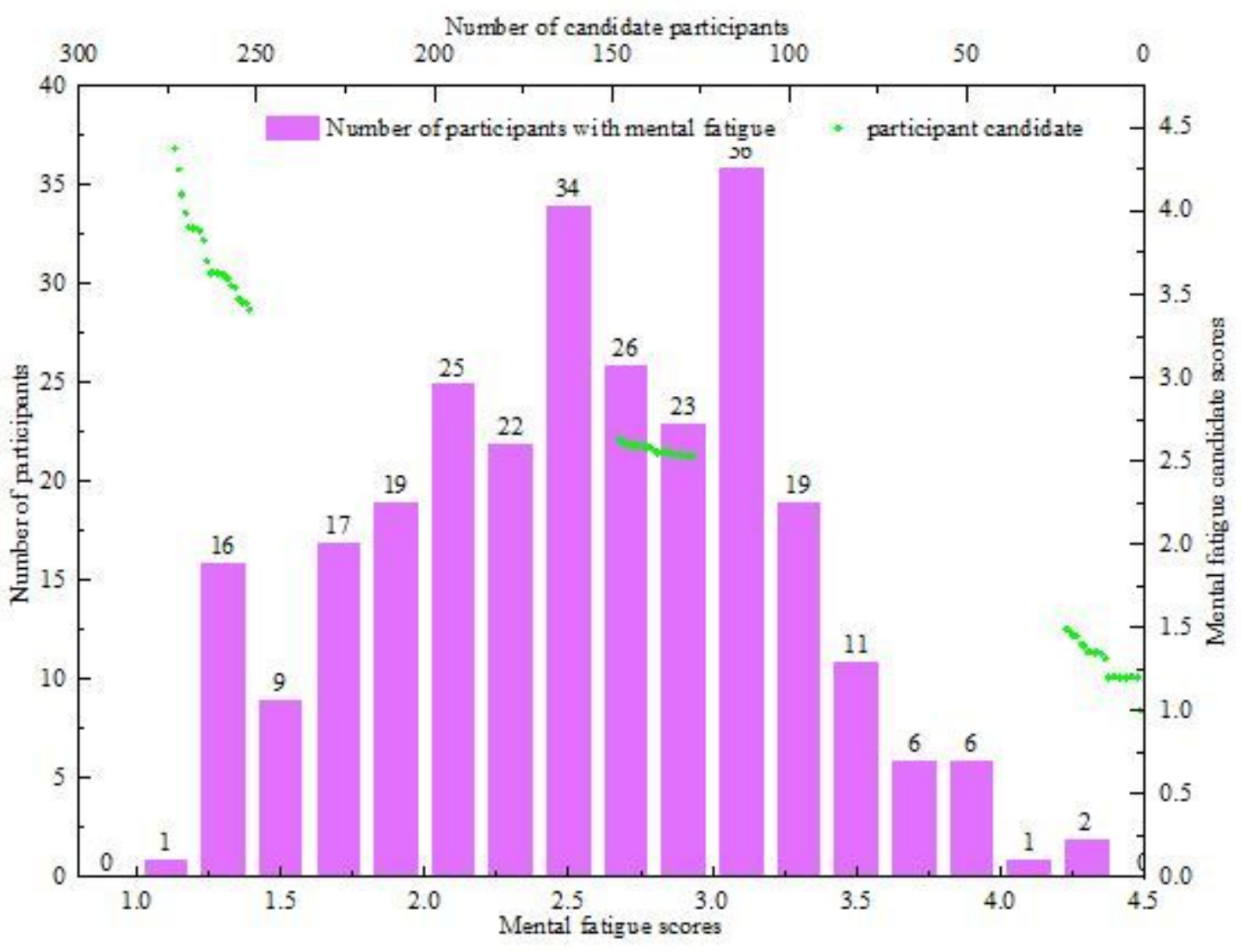

\section{Figure 1}

Miners' mental fatigue scores and participant candidates' statistical chart 


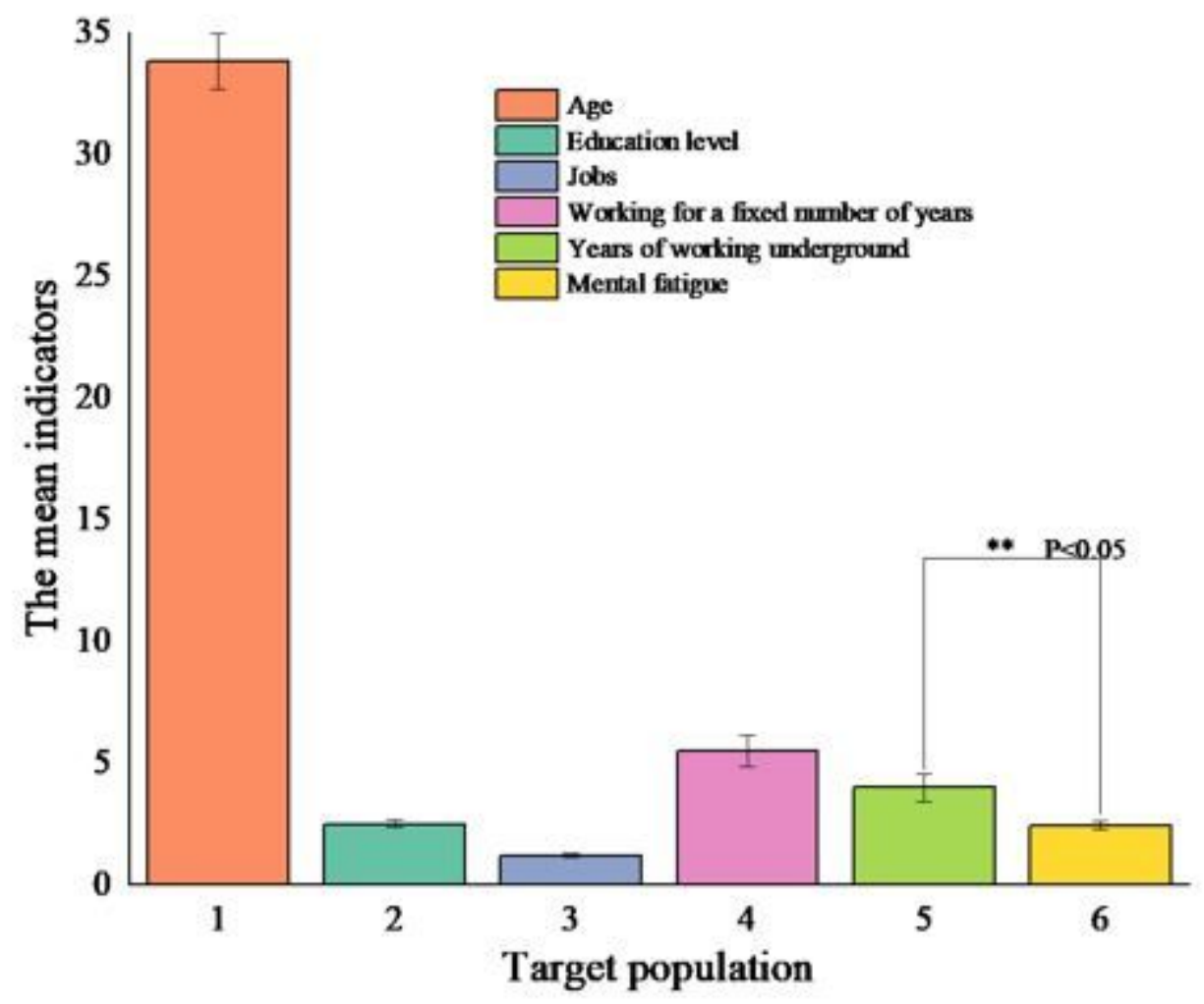

Figure 2

The results of correlation analysis between demographic variables and mental fatigue 


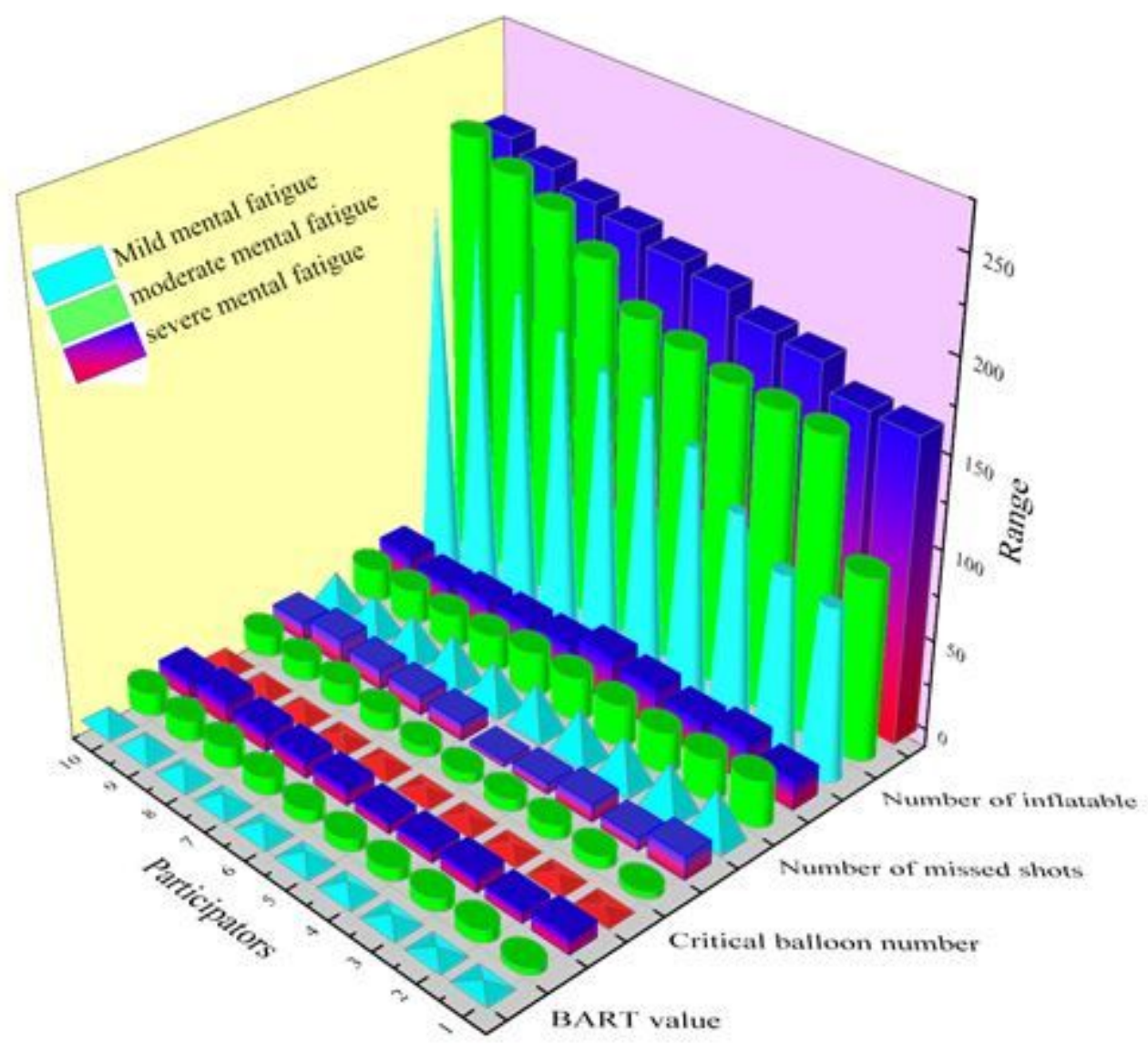

Figure 3

Statistical chart of BART experimental indicators

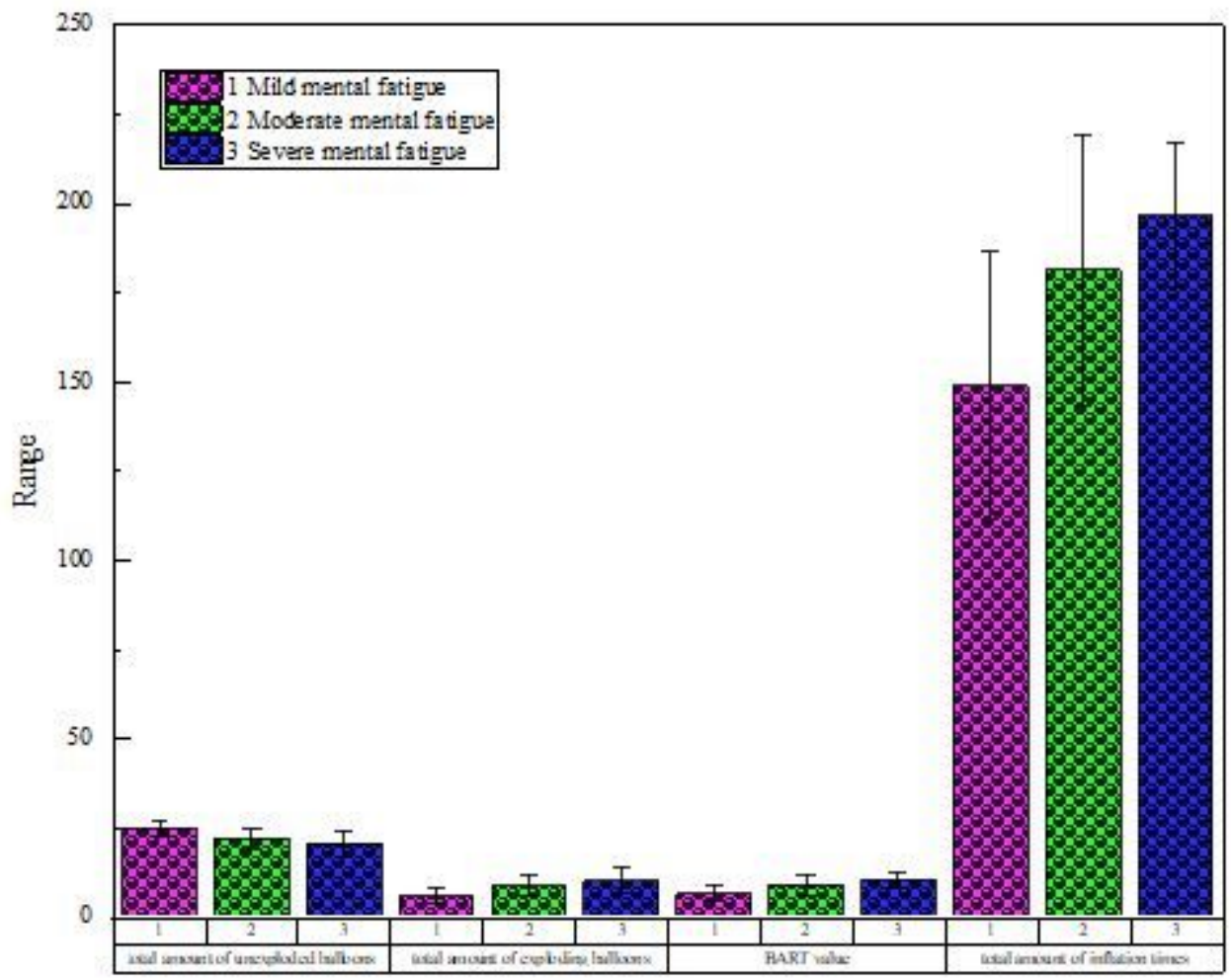


Figure 4

BART index evaluation in different mental fatigue groups $(M \pm S E)$.

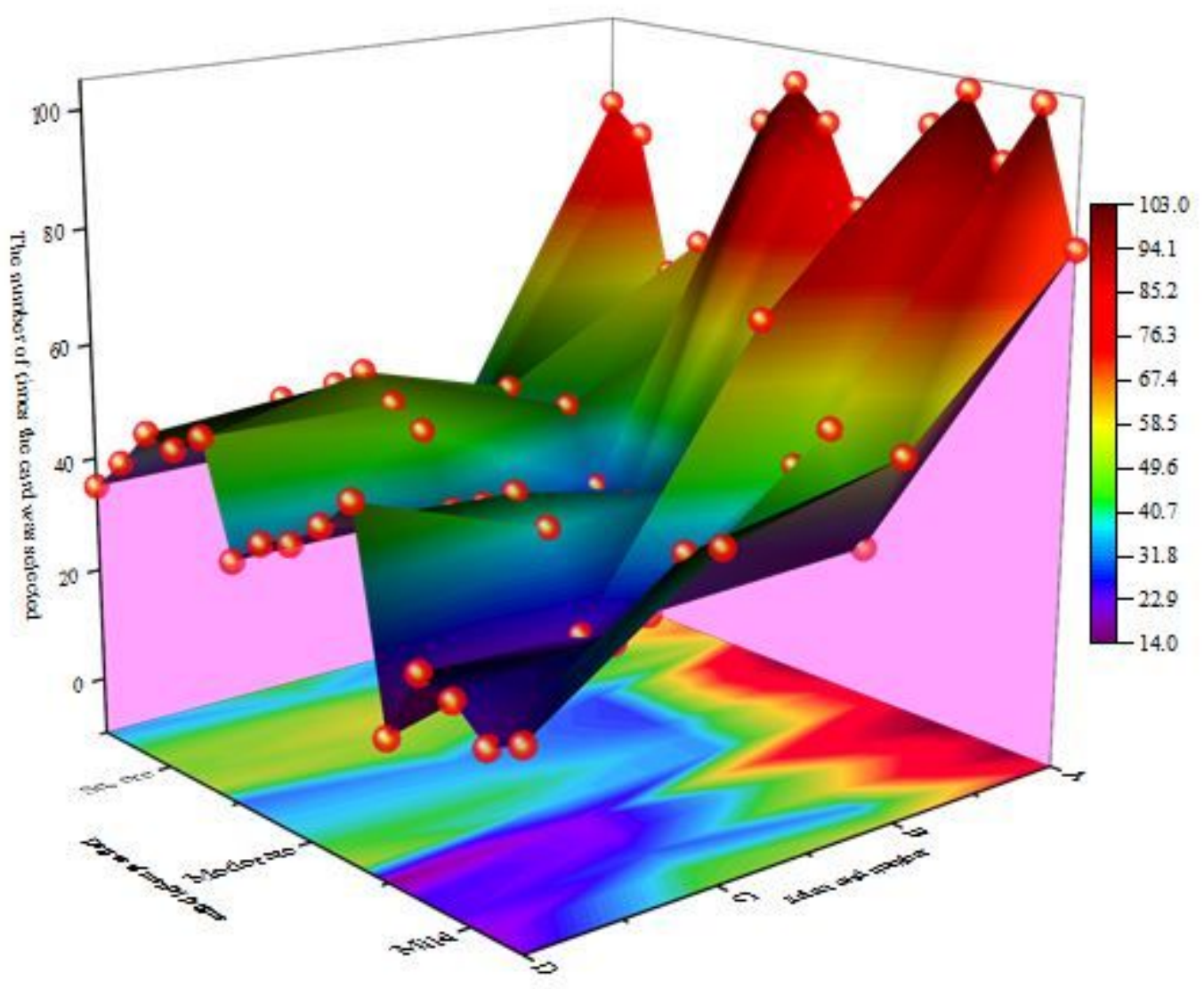

Figure 5

Number of cards selected for different levels of mental fatigue 


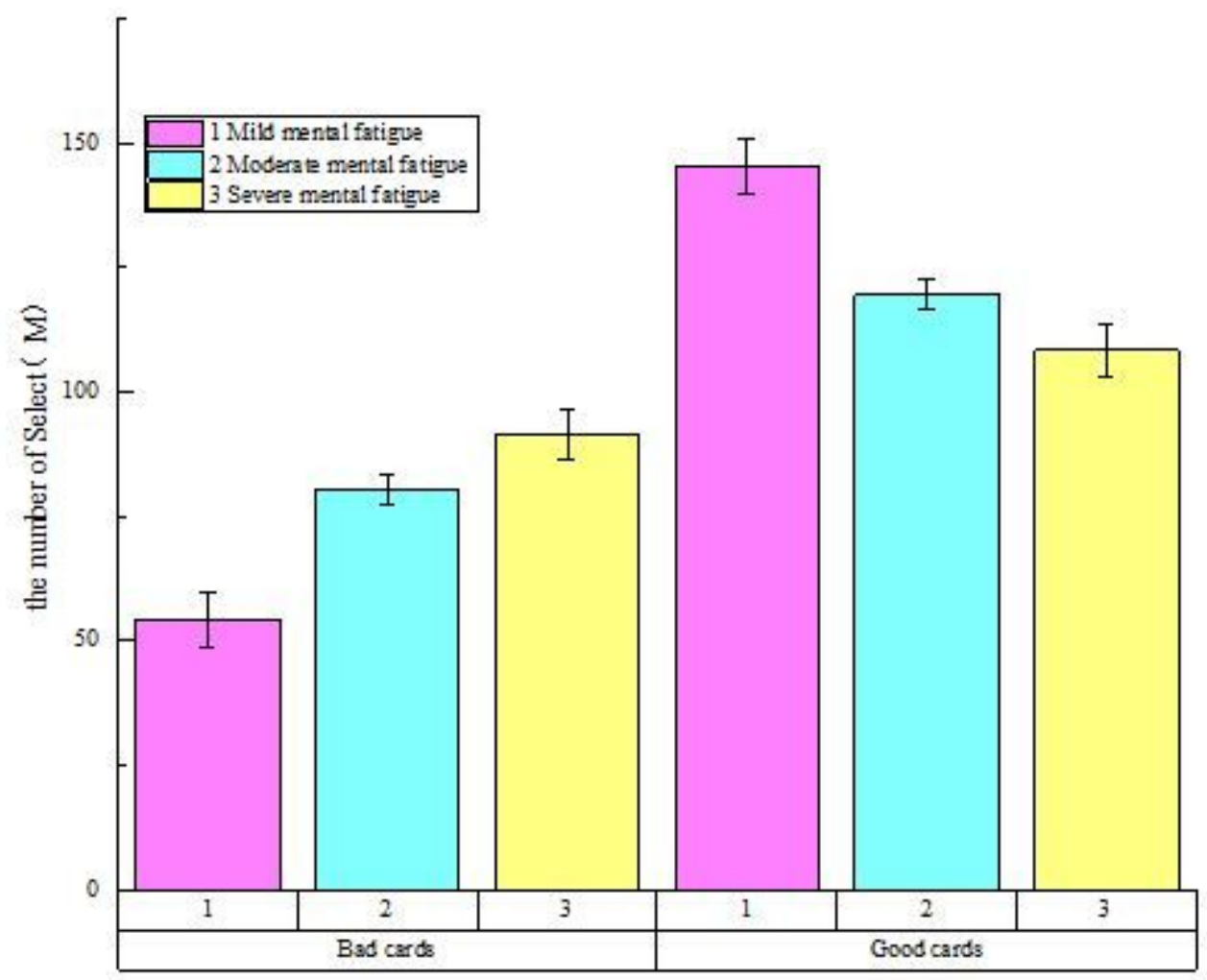

Figure 6

Statistical chart of favorable and unfavorable card selection (M $\pm S E)$. 


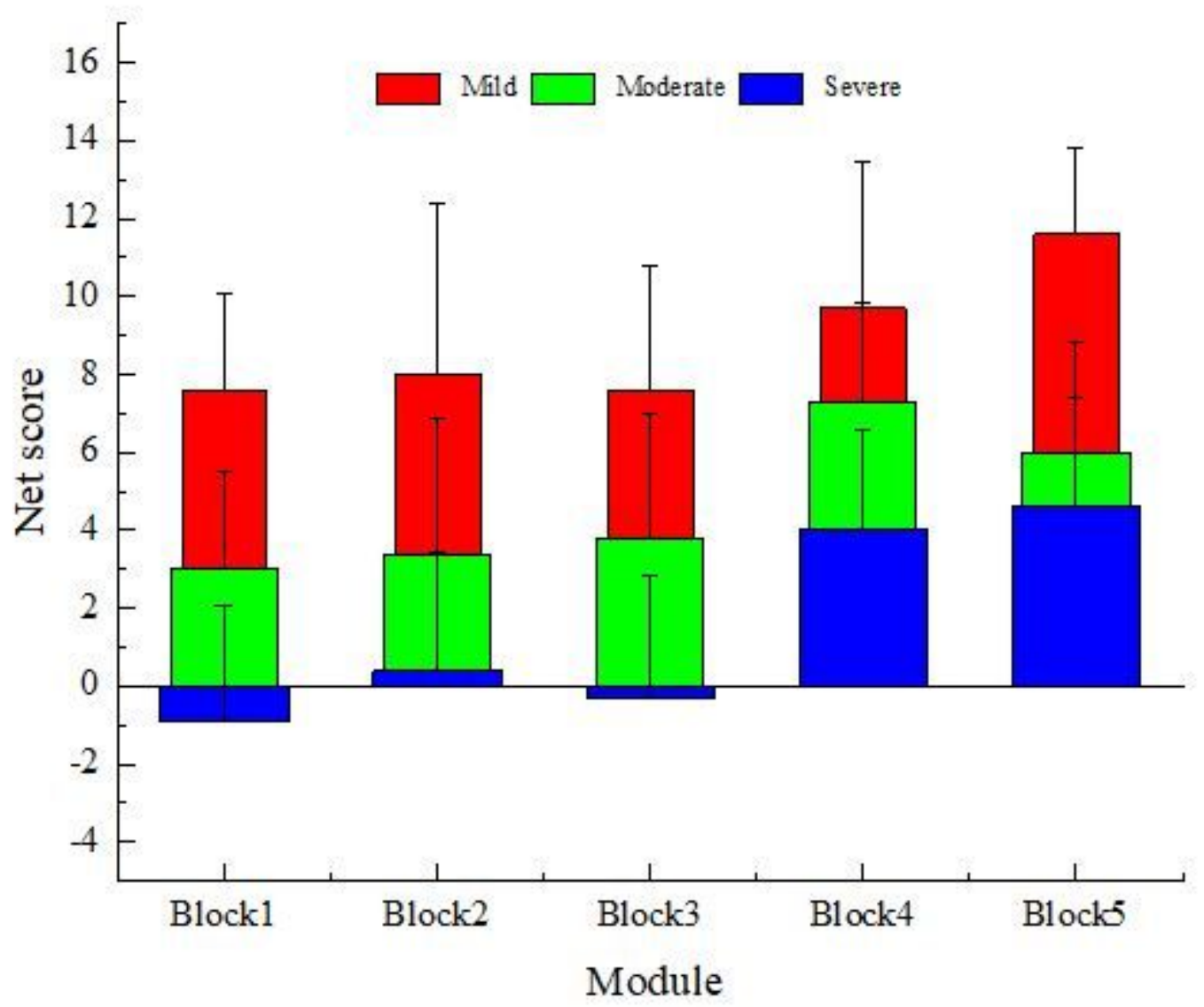

Figure 7

Statistical chart of net score of different modules $(M \pm S E)$ 